\title{
Compositional Stage Mapping by EPMA and micro-XRF
}

\author{
P. K. Carpenter ${ }^{1}$ \\ ${ }^{1}$ Dept. of Earth and Planetary Sciences, Washington University in St. Louis, Campus Box 1169, Saint \\ Louis, MO, 63130, USA
}

The techniques of electron-probe microanalysis (EPMA) and micro-X-ray fluorescence ( $\mu \mathrm{XRF}$ ) are used in complementary ways to analyze samples. Wide area mapping is achieved by driving the stage while using a fixed electron beam or X-ray source to collect a strip of data at a time. EPMA has superior image and analytical spatial resolution especially coupled with the energy-dispersive spectrometer (EDS), but mapping of large samples presents sample preparation and acquisition issues. Samples analyzed by EPMA must be flat, polished, and require carbon coating. Mapping is performed by defocusing the electron beam to match the stage step size. A high probe current is necessary to compensate for short dwell times, and the dose current $*$ time as nA-sec is used to compare with conventional spot analysis times that are 2-3 orders of magnitude longer than map dwell times.

For this study, EPMA mapping runs were made on the WUSTL JEOL JXA-8200 with 5 wavelength-dispersive spectrometers (WDS) and a silicon-drift EDS, using JEOL and Probe Software applications. Several runs were conducted to evaluate spatial coverage vs. dwell time and detection limit considerations. The X-ray intensity maps were acquired using Probe Image and Calcimage software which performs a full $\Phi(\rho z)$ correction at each pixel in the map. The calibration uses a conventional quantitative analysis standardization, and the mean atomic number (MAN) background correction is used to subtract WDS backgrounds. The MAN background correction allows all counting time to be spent on the analytical peak, which significantly improves sensitivity during map runs. Twostage map passes were used to generate a 10 element WDS compositional map. Maps were typically run overnight and map correction required 2-4 hours of computer time.

The WUSTL EDAX Orbis $\mu \mathrm{XRF}$ was used to compare with EPMA mapping. The $\mu \mathrm{XRF}$ has a 30 micron spot size defined by a polycapillary optic, which improves low energy X-ray detection and reduces sampling of X-rays from deep within the sample. The Orbis has a silicon drift EDS detector and generates a spectrum image data cube which can be used to mine data. The map image resolution is defined using the 30 micron spot size, map size, and degree of sampling by the 30 micron beam. Typical compositional maps are acquired with a similar pixel resolution compared to EPMA, with dwell times of 50-500 msec. The $\mu$ XRF can be used to map larger samples which benefit from being polished but do not need to be carbon coated. Map runs typically take hours to days to complete.

A comb-layered xenolith is used to compare compositional mapping by EPMA and $\mu$ XRF. This sample is a 5x6 $\mathrm{cm}$ rock slab containing multiple layers of mm-length dendritic plagioclase, sector-zoned Ca-pyroxene, euhedral to granular olivine, and quenched basaltic glass. It represents directional solidification during rapid cooling from a basaltic melt. Quantitative EPMA stage maps were acquired on a 1-inch round polished section using a $2.25 \mathrm{~mm}$ square map area at $800 \times 800$ pixels, with a $3 \mu \mathrm{m}$ stage step size and beam diameter, dwell time of $150 \mathrm{msec}$ per pixel, and analytical conditions of $15 \mathrm{kV}$ and $250 \mathrm{nA}$. The WDS maps were processed as outlined above. The results are summarized in Fig. 1, which shows BSE, and maps for major and trace elements . The Ca-pyroxene sector zoning is a challenge to map as it exhibits a Cr variation of $\sim 2000-5000 \mathrm{ppm}$. The analytical conditions of 37.5 nA-sec (i.e., $250 \mathrm{nA} * 0.15 \mathrm{sec}$ ) were necessary to measure Cr above the detection (DL) limit; a conventional analysis at $50 \mathrm{nA}$ and $60 \mathrm{sec}$ count time is $300 \mathrm{nA}-\mathrm{sec}$ for comparison. The Calcimage DL map is useful for evaluating the compositional dependence of the DL due to different average $\mathrm{Z}$ of the phases. For the sector zoning there is a difference of 1220-1230 ppm DL for the two zones. The quantitative compositional maps can be further processed and examples will be shown during the presentation.

The $\mu \mathrm{XRF}$ was used to map a $21 \times 12 \mathrm{~mm}$ area on the comb-layer xenolith slab. This area is about an order of magnitude larger compared to the EPMA maps, and similar X-ray maps were produced. Because the $\mu \mathrm{XRF}$ uses 
an EDS detector, one must inspect the EDS spectrum for pulse-pileup artifacts at higher count rates. Additionally, samples with large crystalline grains can cause X-ray diffraction peaks to be present which masquerade as characteristic peaks and appear in maps as a hot region. However, the $\mu \mathrm{XRF}$ has very good sensitivity for higher energy elements such as Sr when using the Ka line for analysis and mapping. Figure 2 illustrates the sum spectrum for a region at the top of the plagioclase layer that has elevated $\mathrm{Sr}$ content. This higher $\mathrm{Sr}$ content is evident in the $\mu$ XRF map which uses the $\mathrm{Sr} \mathrm{K} \alpha$ line and samples a larger area, but was not observed by EPMA which necessarily uses the Sr L $\alpha$ line. This underscores the need to evaluate samples on multiple spatial scales when considering the compositional variations. In summary, both techniques have important advantages for compositional mapping and provide complementary data.
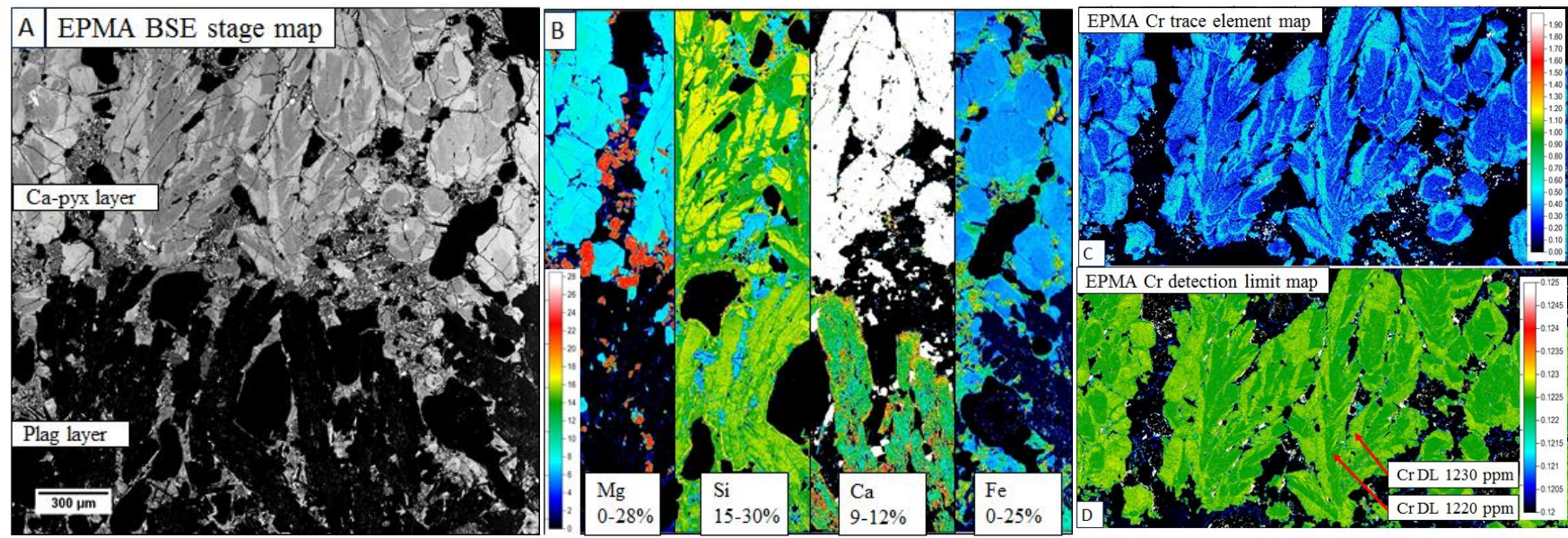

Figure 1. EPMA maps of comb-layer xenolith, all images from $2.25 \mathrm{~mm}$ square map area with 300 micron scale bar. (A) BSE stage map, sector-zoned Ca-pyroxene layer contains granular olivine, plagioclase layer has interstitial glass. (B) Quantitative concentration maps, color scale shown for $\mathrm{Mg}$ and range of element in wt\% shown on $\mathrm{Mg}, \mathrm{Si}, \mathrm{Ca}$, and $\mathrm{Fe}$ maps. Maps are in equal position to BSE map shown in (A). (C) Quantitative concentration map for Cr in Ca-pyroxene layer (upper half of BSE image (A)). Two sector compositions have 2000-5000 ppm concentration. Lower image shows $\mathrm{Cr}$ detection limit of $\sim 1220 \mathrm{ppm}$ at 3-sigma with avg-Z dependence due to major-element sector chemistry.
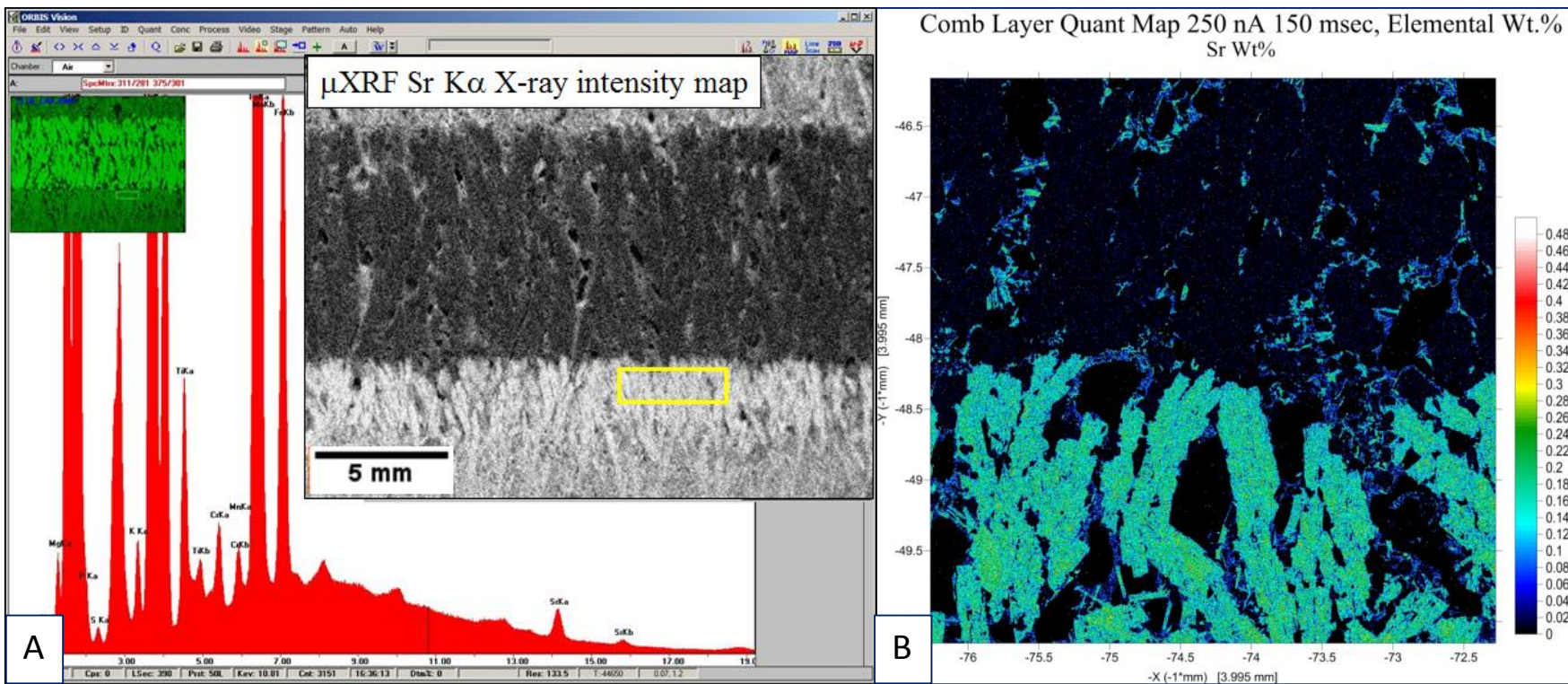

Figure 2. (A) Map from $21 \times 12 \mathrm{~mm}$ area of polished slab from same xenolith using $\mu$ XRF. EDS sum spectrum is derived from yellow region at top of plagioclase layer, and has peaks for both $\mathrm{Sr} K \alpha$ and $\mathrm{K} \beta$. The $\mu \mathrm{XRF}$ map was acquired at $512 \times 400$ pixels, $41 \mu \mathrm{m}$ step size and $500 \mathrm{msec}$ per pixel, and $600 \mu \mathrm{A}$ tube current at $30 \mathrm{kV}$. (B) Quantitative EPMA map for Sr which indicates the Sr level is approximately $2000 \mathrm{ppm}$. 